\title{
Update on radionuclide therapy in oncology (Review)
}

\author{
CORNELIA NITIPIR ${ }^{1,2}$, DANA NICULAE ${ }^{3}$, CRISTINA ORLOV $^{1}$, MARIA ALEXANDRA BARBU $^{1}$, \\ BOGDAN POPESCU ${ }^{1}$, ANA MARIA POPA ${ }^{1}$, ANCA MIHAELA STOIAN PANTEA ${ }^{2}$, ADINA ELENA STANCIU ${ }^{4}$, \\ BIANCA GALATEANU ${ }^{5}$, OCTAV GINGHINA ${ }^{6}$, GEORGIOS Z. PAPADAKIS ${ }^{7}$, BORIS N. IZOTOV ${ }^{8}$, \\ DEMETRIOS A. SPANDIDOS ${ }^{9}$, ARISTIDES M. TSATSAKIS ${ }^{10}$ and CAROLINA NEGREI ${ }^{11}$
}

${ }^{1}$ Oncology Department, Elias University Emergency Hospital, 'Carol Davila' University of Medicine and Pharmacy, 011461 Bucharest; ${ }^{2}$ Hygiene Department, 'Carol Davila' University of Medicine and Pharmacy, 050463 Bucharest;

${ }^{3}$ Radiopharmaceuticals Research Centre, Horia Hulubei National Institute for Physics and Nuclear Engineering, 077125 Magurele; ${ }^{4}$ Department of Carcinogenesis and Molecular Biology, Institute of Oncology, 022328 Bucharest; ${ }^{5}$ Department of Biochemistry and Molecular Biology, University of Bucharest, 050095 Bucharest; ${ }^{6}$ Department of Surgery, Faculty of Dental Medicine, 'Sf. Ioan' Clinical Emergency Hospital, 'Carol Davila' University of Medicine and Pharmacy, 042122 Bucharest, Romania; ${ }^{7}$ Foundation for Research and Technology Hellas (FORTH), Institute of Computer Science (ICS), Computational Biomedicine Laboratory (CBML), 71003 Heraklion, Greece; ${ }^{8}$ Department of Analytical Toxicology, Pharmaceutical Chemistry and Pharmacognosy, Sechenov University, 119991 Moscow, Russia; ${ }^{9}$ Laboratory of Clinical Virology, and ${ }^{10}$ Department of Toxicology and Forensic Sciences, University of Crete Medical School,

71003 Heraklion, Greece; ${ }^{11}$ Departament of Toxicology, Faculty of Pharmacy, 'Carol

Davila’ University of Medicine and Pharmacy, 020956 Bucharest, Romania

Received June 15, 2017; Accepted September 21, 2017

DOI: $10.3892 / \mathrm{ol} .2017 .7141$

\begin{abstract}
Unstable isotopes and their capacity to emit ionizing radiation have been employed in clinical practice not only for diagnostic, but also for therapeutic purposes, with significant contribution in several fields of medicine and primarily in the management of oncologic patients. Their efficacy is associated with their ability to provide the targeted delivery of ionizing radiation for a determined duration. These compounds can be used for curative or palliative treatment, as well as for a diagnostic-therapeutic (theranostic) approach. This review summarises the most recent trends in radionuclide treatment for several malignancies, including prostate cancer, neuroendocrine tumours, and hematological and thyroid malignancies, in which radionuclide-based therapies have been employed with high effectiveness.
\end{abstract}

\section{Contents}

1. Introduction

2. Radionuclide therapy for different types of cancer

3. Conclusions

Correspondence to: Dr Dana Niculae, Radiopharmaceuticals Research Centre, Horia Hulubei National Institute for Physics and Nuclear Engineering, 401A Atomistilor Street, 077125 Magurele, Romania

E-mail: sciencecontactemail@gmail.com

Key words: radionuclide, particle, lutetium, yttrium, iodine, $\beta$-emitting

\section{Introduction}

Nuclear medicine consists of using two major classes of isotopes for therapeutic purposes: Stable ones, not undergoing radioactive decay in time and unstable ones. Stable isotopes are mainly used as tracers in pharmacokinetic studies, in order to investigate biochemical pathways in humans. Stable isotopes already play an important role in current medical research, with great future research applications, since the customised synthesis of novel carbon-13, nitrogen-15 and oxygen-18 radiolabelled compounds, as well as noble gas isotopes are actively expanding (1). Unstable isotopes have an excess of neutrons that interact with the protons in the nucleus, which explains not only their capacity to emit ionizing radiation, but also their decay, measured through their half-life (1). The stability of radioisotope nuclei is typically achieved by an $\alpha$ and/or an electron or positron emission, accompanied by energy emission materialised as gamma-rays (electromagnetic radiation).

The present study is a review of radionuclide therapy in oncology, highlighting the new trends in this field. Approximately 3,800 radiation emitting isotopes can be produced artificially through neutron activation in a nuclear reactor, or by other nuclear reaction in a cyclotron or linear accelerator (2); about 200 radioisotopes have been investigated for potential medical applications and less than 50 are used clinically on a regular basis (3). Globally, there is high prevalence of radioisotope use for diagnostic purposes (ca. 90\%), common practice in more than 10,000 hospitals. More specifically, in $26 \%$ of the world's population, representing the total approximate number of inhabitants of developed countries, diagnostic nuclear medicine procedures are used with a 


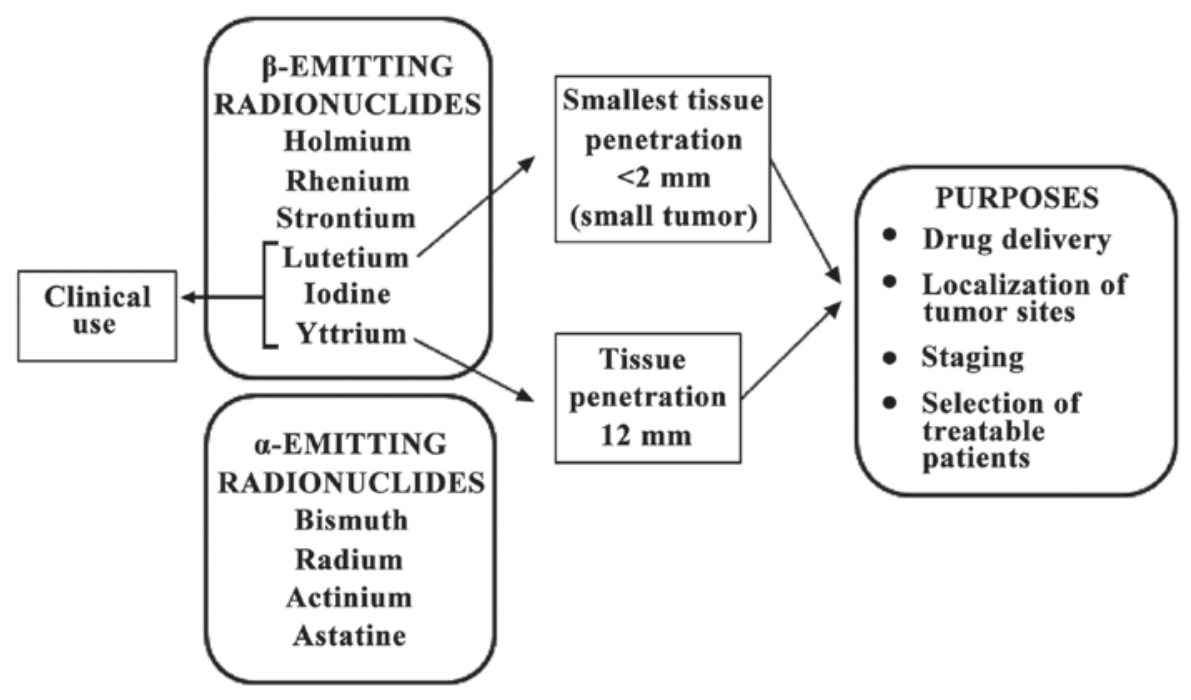

Figure 1. Types of radionuclides and their characteristics.

$1.9 \%$ frequency per year, of which radioisotope therapy represents more or less one tenth. Over 20 million nuclear medicine procedures in 311 million individuals are carried out in the US per year, whereas about 10 million are performed in Europe in 500 million individuals $(4,5)$. Australia is the top country using nuclear medicine for therapeutic purposes (it is estimated that half the current population will be exposed to nuclear radiation for different disease therapies) (6).

\section{Radionuclide therapy for different types of cancer}

Current clinical and preclinical research targeting internal radiotherapy for oncology purposes involves at least $13 \beta$-emitting radionuclides $\left({ }^{177}\right.$ Lutetium, ${ }^{166}$ Holmium, ${ }^{186}$ Rhenium, ${ }^{188}$ Rhenium, ${ }^{67}$ Copper, ${ }^{149}$ Promethium, ${ }^{199}$ Gold, ${ }^{77}$ Bromine, ${ }^{153}$ Samarium, ${ }^{105}$ Rhodium, ${ }^{89}$ Strontium, ${ }^{90}$ Yttrium and ${ }^{131}$ Iodine) and four $\alpha$-emitting radionuclides $\left({ }^{213}\right.$ Bismuth, ${ }^{223}$ Radium, ${ }^{225}$ Actinium and ${ }^{211}$ Astatine) (7-9), characterised by different ranges, distance of effectiveness and relative biologic efficacy (RBE). Notwithstanding this number, the full implementation of targeted radiopharmaceutical therapeutics still suffers from a shortage of radionuclides for research and clinical trials, the more so as of the above radionuclides, only ${ }^{90} \mathrm{Y}$ and ${ }^{131} \mathrm{I}$ are readily available in a form suitable for clinical trial use. These are used in association with monoclonal antibodies in the treatment of non-Hodgkin's lymphoma, whereas samarium-153-EDTMP and strontium-89-chloride are included in the palliation of bone metastases. ${ }^{177}$ Lutetium is the radionuclide with the most attractive physical properties for oncology due to its emission characteristics $(0.5 \mathrm{MeV}$ maximum energy $\beta$ emission and $<2 \mathrm{~mm}$ tissue penetration) permit the energy to focus on the tumour rather than on the surrounding healthy tissue. In comparison to ${ }^{90} \mathrm{Y}$, whose particle range is $12 \mathrm{~mm}$, this is more suitable for small tumours (Fig. 1).

Current clinical practice is characterised by the immediate and sharp need for $\alpha$-emitting therapeutic radionuclides, featuring higher (ca. $100 \mathrm{keV} / \mu \mathrm{m}$ ) linear energy transfer (LET) and shorter action range, able to yield much more selective and localised cytotoxicity. In addition to $\alpha$-emitters, the variety of available theranostics (isotope compounds allowing for both imaging and therapy) needs to include $\beta$-emitters for improved determination of the radiation dose (10).

As far as therapy is concerned, the suitability of a particular radionuclide primarily relies on radionuclide properties, both physical and chemical, on manufacturing methods, as well as on biological behaviour (the more so if in vivo dissociation from the carrier molecule is involved); however, other additional aspects, such as specific activity, radionuclide purity, target nuclide occurrence in the environment, feasibility and ease of radionuclide production in a suitable form for application should not be overlooked either (11). In addition, the efficacy of radionuclide therapies could be further enhanced by implementing fractionated dose radiotherapy schemes, combining radiopharmaceutical with radio-sensitizing medication, or adopting pre-targeting radionuclide strategies (pRIT). Molecular imaging probes function by the delivery of radionuclides to tumour tissue directed to antibodies or peptides, targeting specific malignancy-related biochemical pathways enabling non-invasive imaging at molecular level. Since biochemical alterations occur at an earlier stage compared to the detection of anatomical abnormalities, molecular imaging probes allow the efficient early-stage localization of tumour sites, accurate disease staging and restaging, tracking of drug effectiveness, systemic treatment of all disease sites, monitoring of treatment response and the selection of patients suitable for radionuclide-based therapies, facilitating personalised diagnostic and treatment strategies (12). In systemic radiotherapy, radiolabelled molecular probes targeting the same biochemical processes deliver in situ high destructive capacity of $\beta$ - and $\alpha$-emitting radionuclides, which destroy cancer cells by damaging their DNA. This has been proven to be effective for the treatment of several malignancies, enabling the eradication of disseminated tumour cells and small metastases (13).

Accounting for $15 \%$ of all malignancies typically diagnosed in males (14), prostate cancer ranks the most frequent cancer affecing males worldwide, resulting in significant morbidity and mortality. Although usually presenting in early stages, the number of patients with metastases at diagnosis is important. Radical prostatectomy and external beam radiotherapy are used for treatment of local disease, whereas androgen depri- 
vation therapy or chemotherapy are the available choices for metastatic stages (15).

Prostate specific membrane antigen (PSMA) is a type II transmembrane protein on the surface of neoplastic prostate cells, which currently is the most promising target for both imaging and treatment, since it is upregulated in the majority of cases of prostate cancer, regardless of disease stage. Furthermore, PSMA is internalised following antibody binding, while is not released into the blood stream. PSMA may be encountered in normal tissues as the lacrimal glands, the salivary glands or the small intestine; however, in normal prostatic tissue, PSMA is expressed only in the apical epithelium of secretory ducts at low levels. In recent years, positron emitting compounds that target PSMA have shown great potential for the accurate detection of all disease sites, regardless of the levels of prostate-specific antigen (PSA). In addition, PSMA peptides and antibodies can be conjugated with ${ }^{131} \mathrm{I},{ }^{90} \mathrm{Y}$ or ${ }^{177} \mathrm{Lu}$ for the treatment of metastatic, castrate-resistant prostate cancer. However, for the treatment of advanced prostate cancer, the FDA, for instance, has approved no PSMA targeting radionuclide therapy. This type of peptide most often described in the literature is PSMA-DKFZ-617. Apart from being easily conjugated with peptides, ${ }^{177} \mathrm{Lu}$ has the advantage of a long half-life. Therefore, it irradiates on a very small area, but for a long period of time. In most reported trials, ${ }^{177} \mathrm{Lu}$ is administered at 6-week intervals or longer (16).

A multicentre German trial enrolling 145 patients analysed the efficacy of ${ }^{177} \mathrm{Lu}$ as a therapeutic agent in patients with advanced stage prostate cancer. The patients were exposed to a maximum of 4 cycles of radioisotope therapy, with a total dosage of $8 \mathrm{GBq}$. The study primary endpoint was the biochemical response, the level of PSA being determined for 16 weeks after treatment, but toxicity was also reported. During treatment or follow-up, 19 patients died, 18 had grade 3 or 4 toxicities reported, among which xerostomia was the most frequent. By the end of follow-up time (16 weeks), $45 \%$ of the patients exhibited a decrease of $\geq 50 \%$ in PSA levels. The response was early in over $40 \%$ of the patients, usually after the first course (17).

A different trial enrolling 82 patients reported similar response rates to only one administration of isotope-based therapy. The biochemical response rate (defined as an $>50 \%$ decrease in PSA levels ) was $31 \%$. Furthermore, $47 \%$ displayed a $25-50 \%$ decrease in PSA levels and in another $23 \%$ of patients, PSA levels increased by <25\% (18).

Thus, radiolabelled PSMA ligands hold promise for the management of patients with metastatic prostate cancer, enabling accurate imaging, disease staging, restaging and therapy, facilitating personalised treatment strategies.

Radionuclide therapy has also been employed in the management of patients diagnosed with neuroendocrine tumours (NETs). Localised NETs are treated with surgical excision. However, $>40 \%$ of patients with NETs present with metastatic disease on diagnosis, requiring the application of systemic treatment strategies. Gastrointestinal NETs have been investigated with ${ }^{177}$ Lu-DOTATATE scintigraphy for some time now, enabling the detection of metastatic sites that were not visible by means of anatomical imaging techniques. Furthermore, this investigation informs on the tumour affinity for this isotope, revealing patients with a high likelihood to respond positively. The phase III, randomised NETTER-1 trial revealed remarkable tolerability and efficiency for ${ }^{177}$ Lu-DOTATATE. The study enrolled 230 patients with midgut, metastatic NETs already receiving long acting release-LAR octreotide (19).

The enrolled patients were randomly assigned to receive ${ }^{177} \mathrm{Lu}$-DOTATATE combined with LAR octreotide or LAR octreotide alone. The final results have not yet been published, but intermediate analysis of the data shows a clear advantage for isotope therapy in progression-free survival (PFS). The LAR octreotide arm has reached this endpoint, with an 8.4 months PFS. The fact that such PFS is rarely achieved in cancer holds great promise for the role of PRRT (peptide receptor radionuclide therapy) in (neo)adjuvant management of patients with NETs (19).

As previously mentioned (3), ${ }^{90} \mathrm{Y}$ is another option for isotope-based treatments. Not only does it emit the highest levels of $\beta$ energy with a maximum of $2.28 \mathrm{MeV}$, but it also has important tissue penetration: $12 \mathrm{~mm}$, which is why it should be administered locally.

SIR-spheres, the radioactive units that can be used for selective internal radiation therapy in hepatic metastases or primary tumours, contain ${ }^{90} \mathrm{Y}$. For colorectal cancer (CRC) with liver metastases, they have been tested alone, in comparison to chemoembolisation, or together with systemic therapy (20).

The efficacy and toxicity profiles of the combined approach are just beginning to be studied. The SIRFLOX study enrolled 530 patients with CRC and unresectable liver metastases. They were randomised to first-line treatment with FOLFOX VI (bevacizumab at investigator's discretion) alone or with SIR-spheres. The median PFS was 10.7 months in the SIR-spheres arm vs. 10.2 in the standard of care arm. There was a significantly longer median duration of liver PFS in a completing risk analysis (20.5 vs. 12.6 months; HR, 0.69; 95\% CI, 0.55-0.90). There was no significant improvement in the rate of subsequent liver resection in the SIR group. Worse toxicities more common with SIR-spheres were neutropenia ( $41 \%$ as compared to $29 \%$ ), febrile neutropenia (6\% as compared to $2 \%$ ), thrombocytopenia (10\% as compared to $3 \%$ ), gastric or duodenal ulcer (4\% as compared to $0 \%$ ), and ascites (23\% as compared to $0 \%$ ). One also has to take into account that that team needed for SIR-spheres administration consists in a wide variety of medical specialties and the procedure itself is expensive and long-lasting. Therefore, until further research is undertaken, radioembolisation is not a standard first line-therapy, but can be taken into account for later lines of therapy (20).

As opposed to conventional chemotherapy, radioimmunotherapy enables specific targeting of malignant cells and delivery of monoclonal antibodies. This strategy has been proven to be efficient in the treatment of low-grade B-cell non-Hodgkin lymphoma, by using ${ }^{90} \mathrm{Y}$ or ${ }^{131} \mathrm{I}$ marked monoclonal antibodies: ${ }^{90}$ Y-ibritumomab tiuxetane and ${ }^{131}$ I tositumomab. Tositumomab has high affinity for the CD20 receptor on the B lymphocyte surface, either malignant or normal. When both these agents reach their target, they not only have the classical monoclonal antibody effect, but also deliver ionizing radiation to the cell $(21,22)$.

The same strategy was used for pancreatic cancer. ${ }^{90}$ Y-clivatuzumab is a complex targeting the hPAM4 antigen that resides on pancreatic ductal malignant cells only. This complex 
was administered together with gemcitabine in a small dosage in 38 patients with locally-advanced and metastatic pancreatic cancer enrolled in a phase II clinical trial. Gemcitabine was administered for 4 weeks at a dosage of $200 \mathrm{mg} / \mathrm{m}^{2}$. The isotope-antibody complex had three administrations, in weeks 2, 3 and 4. In 28 patients, grade 3 or 4 thrombocytopenia was reported, although it is thought that fractionation contributed to a lower toxicity profile. Six patients displayed partial response and stable disease was reported in 16, with 7.7 months PFS, demonstrating the efficacy of this option (23).

One of the first radionuclide therapy alternatives ever used was in cases of papillary or follicular thyroid cancer. The medullary type, lymphoma or anaplastic types do not have the tumour biology necessary to take up the isotope, therefore in these cases such treatment has no purpose. ${ }^{131} \mathrm{I}$ is both a local treatment, providing the ablation of the remaining post-operative tissue and a treatment of the possibly existing metastases. It was repeatedly proven that it prolongs PFS and overall survival, being a highly active treatment (24).

Radioiodine has dose dependent toxicity. A higher incidence of leukaemia and solid malignancies has been reported in the treated population, compared to general one. Due to the relatively low dose delivered to other tissues, only patients exposed to high cumulative ${ }^{131} \mathrm{I}$ activities $(>600 \mathrm{mCi})$ are considered at risk. However, in the case of a patient with functioning thyroid metastases, these doses can be necessary (25). This is why the need to separate responders from non-responders to radioiodine is a pending issue. A mathematical model has recently been established for treatment individualisation. A clinical trial with this purpose enrolled 50 patients initially treated with total thyroidectomy associated with lymph node dissection and subsequent radiation therapy. The criteria for patient classification as responders or non-responders were decrease of number of RAI-avid foci and the absence of new lesions on post-therapy body scan and, most importantly, decrease in stimulating thyroglobulin $(\mathrm{Tg})$ over time. Tumour doubling time $\left(\mathrm{T}_{\mathrm{d}}\right)$ was an additional important parameter. This study shows usefulness of mathematical modelling in clinical decision-making and delivery of personalised care $(26,27)$.

Head and neck cancers can also benefit from radionuclide-related procedures, both diagnostic and therapeutic. One example is passive nanotargeting delivery of radionuclides for tumour radiotherapy. This concept was first used for tumour imaging. Radiolabelled pegylated liposomes used to target solid tumours also proved useful for delivery of $\beta$-emitting radionuclides, especially in head and neck patients (28).

However, several new detection methods have been imagined for cancer detection in the early stages. Colorimetric, narrow band imaging, video contact endoscopy and SPIES software filters represent a category of so-called optical biopsy that help the surgeon to early detect and define the tumour margins for free disease resection margin (29-33). ELISA and flow cytometric analysis allow the evaluation of tumour cells, which can influence the prognosis (34).

Scattered, painful bone metastases often bring about poor quality of life in breast cancer patients. The only option in most cases is energetic, however for the price of toxicity and dependence. Radiopharmaceuticals, such as ${ }^{186}$ Re-hydroxyethylidene diphosphonate (HEDP) are an attractive alternative. The overall response rate to this compound is $>70 \%$. Given its short half-life, bone marrow toxicity is low in this case. Choosing a more aggressive, longer half-life compound could generate bone marrow suppression in a heavily pre-treated patient. An additional advantage is its easy, safe administration that does not require specialised personnel; therefore, this option should be taken into account whenever the clinical situation permits (35).

\section{Conclusions}

In conclusion, ${ }^{131} \mathrm{I}$ is no longer the only efficient radionuclide therapy in oncology and a new, promising era of novel malignancy specific radiopharmaceuticals is actively emerging potentially improving the management and outcomes for oncologic patients. Highly specific and more sophisticated strategies such as radioimmunotherapy increase the treatment arsenal, paving the way towards individualised medicine. However, the full potential of this field is far from being completely exploited. Randomised double-blinded multicentre prospective studies are needed in order to elucidate potential contribution to therapeutic efficacy of radionuclide therapies in comparison to conventional therapeutic schemes.

\section{References}

1. Stable isotopes: http://chemistry.tutorvista.com/inorganicchemistry/stable-and-unstable-isotopes.html. Accessed April 16 2017.

2. Nuclear Medicine: Medical isotopes: General concepts https://www.radiochemistry.org/nuclearmedicine/radioisotopes/01_ isotopes.shtml\#top. Accessed April 16, 2017.

3. American Nuclear Society: Medical use of radioisotopes, 2014. www.nuclearconnect.org.

4. Australian Nuclear Science and Technology Organisation: What are radioisotopes? http://www.ansto.gov.au/NuclearFacts/ AboutNuclearScience/Radioisotopes. Accessed April 16, 2017.

5. World Nuclear Association: Radioisotopes in medicine. http:// www.world-nuclear.org/information-library/non-power-nuclearapplications/radioisotopes-research/radioisotopes-in-medicine.aspx (Updated December 2016). Accessed April, 2017.

6. Zalutsky MR: Radionuclide therapy. In: Handbook of Nuclear Chemistry: Radiochemistry and Radiopharmaceutical Chemistry in Life Sciences. Roesch F (ed). Vol 4. Kluwer Academic, Dordrecht, pp315-348, 2003.

7. Harris TJR, Kalen JD and Hall J: Report of meeting held to discuss existing and future radionuclide requirements for the national cancer institute. https:/www.isotopes.gov/outreach/ reports/Radionuclide_Report.pdf.

8. Nordberg E, Orlova A, Friedman M, Tolmachev V, Ståhl S, Nilsson FY, Glimelius B and Carlsson J: In vivo and in vitro uptake of ${ }^{111} \mathrm{In}$, delivered with the affibody molecule $\left(\mathrm{Z}_{\mathrm{EGFR}: 955}\right)_{2}$, in EGFR expressing tumour cells. Oncol Rep 19: 853-857, 2008.

9. Sabongi JG, Gonçalves MC, Alves CD, Alves J, ScapulatempoNeto C and Moriguchi SM: Lutetium 177-DOTA-TATE therapy for esthesioneuroblastoma: A case report. Exp Ther Med 12: 3078-3082, 2016

10. Bobeica M, Niculae D, Balabanski D, Filipescu D, Gheorghe I, Ghita DG and Luo W: Radioisotope production for medical applications at ELI-NP. Rom Rep Phys 68 (Suppl): 847-83, 2016.

11. Cutler CS, Chanda N, Shukla R, Sisay N, Cantorias M, Zambre A, McLaughlin M, Kelsey J, Upenandran A, Robertson D, et al: Nanoparticles and phage display selected peptides for imaging and therapy of cancer. In: Theranostics, Gallium-68 and Other Radionuclides. Recent Results in Cancer Research. Vol 194. Baum RP and Rosch F (eds). Springer, Heidelberg, pp133-147, 2013.

12. National Research Council (US) and Institute of Medicine (US) Committee on State of the Science of Nuclear Medicine: Advancing nuclear medicine through innovation. National Academies Press, Washington, DC, 2007.

13. Kwekkeboom DJ, Mueller-Brand J, Paganelli G, Anthony LB, Pauwels S, Kvols LK, O'dorisio TM, Valkema R, Bodei L, Chinol M, et al: Overview of results of peptide receptor radionuclide therapy with 3 radiolabeled somatostatin analogs. J Nucl Med 46 (Suppl 1): 62-66, 2005. 
14. Emmet L, Willowson K, Violet J, Shin J, Blanksby A and Lee J: Lutetium177 PSMA radionuclide therapy for men with prostate cancer: A review of the current literature and discussion of practical aspects of therapy. J Med Radiat Sci 64: 52-60, 2017.

15. Weill Cornell Medicine: Using radiation, radioimmunotherapy and radioactive isotopes such as lutetium 177 to treat prostate cancer. https://weillcornellgucancer.org/2017/01/12/usingradiation-radioimmunotherapy-and-radioactive-isotopes-such-aslutetium-177-to-treat-prostate-cancer. Accessed April 16, 2017.

16. Ahmadzadehfar H, Rahbar K, Kürpig S, Bögemann M, Claesener M, Eppard E, Gärtner F, Rogenhofer S, Schäfers M and Essler M: Early side effects and first results of radioligand therapy with (177)Lu-DKFZ-617 PSMA of castrate-resistant metastatic prostate cancer: a two-centre study. EJNMMI Res 5 114,2015

17. Rahbar K, Ahmadzadehfar H, Kratochwil C, Haberkorn U, Schäfers M, Essler M, Baum RP, Kulkarni HR, Schmidt M, Drzezga A, et al: German multicenter study investigating ${ }^{177} \mathrm{Lu}$-PSMA-617 radioligand therapy in advanced prostate cancer patients. J Nucl Med 58: 85-90, 2017.

18. Rahbar K, Schmidt M, Heinzel A, Eppard E, Bode A, Yordanova A, Claesener M, Ahmadzadehfar H: Response and tolerability of a single dose of ${ }^{177} \mathrm{Lu}$-PSMA-617 in patients with metastatic castration-resistant prostate cancer: A multicenter retrospective analysis. J Nucl Med 57: 1334-1338, 2016.

19. Strosberg J, El-Haddad G, Wolin E, Hendifar A, Yao J, Chasen B Mittra E, Kunz PL, Kulke MH, Jacene H, et al; NETTER-1 Tria Investigators: Phase 3 trial of ${ }^{177} \mathrm{Lu}$-Dotatate for midgut neuroendocrine tumors. N Engl J Med 376: 125-135, 2017.

20. van Hazel GA, Heinemann V, Sharma NK, Findlay MP, Ricke J, Peeters M, Perez D, Robinson BA, Strickland AH, Ferguson T, et al: SIRFLOX: Randomized phase III trial comparing first-line mFOLFOX6 (plus or minus bevacizumab) versus mFOLFOX6 (plus or minus bevacizumab) plus selective internal radiation therapy in patients with metastatic colorectal cancer. J Clin Oncol 34: 1723-1731, 2016.

21. Weigert O, Illidge T, Hiddemann $\mathrm{W}$ and Dreyling $\mathrm{M}$ : Recommendations for the use of yttrium-90 ibritumomab tiuxetan in malignant lymphoma. Cancer 107: 686-695, 2006

22. Kaminski MS, Tuck M, Estes J, Kolstad A, Ross CW, Zasadny K, Regan D, Kison P, Fisher S, KrollS and Wahl RL: ${ }^{131}$ I-tositumomab therapy as initial treatment for follicular lymphoma. N Engl J Med 352: 441-449, 2005

23. Ocean AJ, Pennington KL, Guarino MJ, Sheikh A, Bekaii-Saab T, Serafini AN, Lee D, Sung MW, Gulec SA, Goldsmith SJ, et al: Fractionated radioimmunotherapy with (90) Y-clivatuzumab tetraxetan and low-dose gemcitabine is active in advanced pancreatic cancer: A phase 1 trial. Cancer 118: 5497-5506, 2012.

24. Tuttle RM: Differentiated thyroid cancer: Radioiodine treatment. Ross DS and Mulder JE (eds). https://www.uptodate. com/contents/differentiated-thyroid-cancer-radioiodine-treatment? source=see_link. Accessed April 18, 2017.
25. Nixon IJ, Ganly I, Patel SG, Palmer FL, Di Lorenzo MM, Grewal RK, Larson SM, Tuttle RM, Shaha A and Shah JP: The results of selective use of radioactive iodine on survival and on recurrence in the management of papillary thyroid cancer, based on Memorial Sloan-Kettering Cancer Center risk group stratification. Thyroid 23: 683-694, 2013.

26. American Thyroid Association (ATA) Guidelines Taskforce on Thyroid Nodules and Differentiated Thyroid Cancer; Cooper DS, Doherty GM, Haugen BR, Kloos RT, Lee SL, Mandel SJ, Mazzaferri EL, McIver B, Pacini F, Schlumberger M, et al: Revised American Thyroid Association management guidelines for patients with thyroid nodules and differentiated thyroid cancer. Thyroid 19: 1167-1214, 2009.

27. Barbolosi D, Summer I, Meille C, Serre R, Kelly A, Zerdoud S, Bournaud C, Schvartz C, Toubeau M, Toubert ME, et al: Modeling therapeutic response to radioiodine in metastatic thyroid cancer: A proof-of-concept study for individualized medicine. Oncotarget 8: 39167-39176, 2017.

28. Harrington KJ, Mohammadtaghi S, Uster PS, Glass D, Peters AM, Vile RG and Stewart JS: Effective targeting of solid tumors in patients with locally advanced cancers by radiolabeled pegylated liposomes. Clin Cancer Res 7: 243-254, 2001.

29. Stefanescu DC, Ceachir O, Zainea V, Hainarosie M, Pietrosanu C, Ionita IG and Hainarosie R: Methilene blue video contact endoscopy enhancing methods. Rev Chim 67: 1558-1559, 2016.

30. Hainarosie R, Ceachir O, Zainea V, Hainarosie M, Pietrosanu C, Zamfir C and Stefanescu DC: The test of lugol iodine solution associated with NBI examination in early diagnostic of tongue carcinoma. Rev Chim 68: 226-227, 2017.

31. Stefanescu DC, Ceachir O, Zainea V, Hainarosie M, Pietrosanu C, Ionita IG and Hainarosie R: The use ofmethylene blue in assessing disease free margins during $\mathrm{CO}_{2}$ LASER assisted direct laryngoscopy for glottis cancer. Rev Chim 67: 1327-1328, 2016.

32. Hainarosie R, Zainea V, Ceachir O, Hainarosie M, Pietrosanu C and Stefanescu DC: The use of methylene blue in early detection of the vocal fold cancer. Rev Chim 68: 16-17, 2017.

33. Stefanescu DC, Ceachir O, Zainea V, Hainarosie M, Pietrosanu C, Ionita IG and Hainarosie R: The value of toluidine blue staining test in assessing disease free margins of oral cavity carcinomas. Rev Chim 67: 1255-1256, 2016.

34. Petrică-Matei GG, Iordache F, Hainărosie R and Bostan M Characterization of the tumor cells from human head and neck cancer. Rom J Morphol Embryol 57 (Suppl): 791-799, 2016.

35. Lam MG, de Klerk JM and van Rijk PP: ${ }^{186}$ Re-HEDP for metastatic bone pain in breast cancer patients. Eur J Nucl Med Mol Imaging 31 (Suppl 1): 162-170, 2004. 